Published in final edited form as:

Expert Rev Obstet Gynecol. 2010 March ; 5(2): 177-181. doi:10.1586/eog.10.12.

\title{
Pregnancy and labor massage
}

\author{
Tiffany Field \\ Touch Research Institute, University of Miami School of Medicine, Department of Pediatrics (D-820), \\ PO Box 016820, Miami, FL 33101, USA
}

\begin{abstract}
Massage therapy has been demonstrated to be effective during pregnancy. Women who received massage therapy reported decreased depression, anxiety, and leg and back pain. Cortisol levels decreased and, in turn, excessive fetal activity decreased, and the rate of prematurity was lower in the massage group. In a study of labor pain, women who received massage therapy experienced significantly less pain, and their labors were on average $3 \mathrm{~h}$ shorter with less need for medication. An underlying mechanism we have been exploring is that these effects are mediated by increased vagal activity. This likely occurs by the stimulation of pressure receptors that are innervated by vagal afferent fibers, which ultimately project to the limbic system, including hypothalamic structures involved in autonomic nervous system regulation and cortisol secretion.
\end{abstract}

\section{Keywords}

alternative therapy; labor pain; postpartum depression; pregnancy massage; prematurity

Complementary and alternative therapies have become increasingly popular for pregnant women and women in labor. In one survey, the majority of pregnant women who participated $(62 \%)$ and $61 \%$ of providers of prenatal healthcare reported that they used complementary and alternative therapies during pregnancy [1]. The most common alternative therapies recommended during pregnancy were massage therapy $(61 \%)$, acupuncture $(45 \%)$, relaxation (43\%), yoga (41\%) and chiropractic therapies (37\%).

\section{Pregnancy massage}

Massage therapy has been demonstrated to be effective during pregnancy in three studies by our group [2-4]. In the first study, therapists provided massage therapy for 20 min per week for 5 weeks [4]. The women reported decreased depression, anxiety and leg and back pain. Cortisol levels decreased and, in turn, excessive fetal activity decreased, and the rate of prematurity was lower in the massage group.

In the second study, depressed pregnant women were massaged by their significant others over a 16-week period using the same protocol (20-min massages twice weekly) [2]. As in the first study, significant decreases were noted in depression and anxiety, as well as cortisol levels. In addition, like the results of the first study, pregnancy outcomes were improved, including a

\footnotetext{
(C) 2010 Expert Reviews Ltd

Tel.: +1 305243 6781, Fax: +1 305243 6488, tfield@med.miami.edu.

Financial \& competing interests disclosure

The author has no other relevant affiliations or financial involvement with any organization or entity with a financial interest in or financial conflict with the subject matter or materials discussed in the manuscript apart from those disclosed.

No writing assistance was utilized in the production of this manuscript.
} 
reduction in excessive fetal movement that has been noted in fetuses of depressed women (Figure 1). The most important finding was the lower incidence of prematurity ( $0 \mathrm{vs} 11 \%$ in the control group).

In the third study, pregnant women diagnosed with major depression were given 12 weeks of twice weekly massage therapy by their significant other, or only standard treatment as a control group [3]. The massage therapy group women versus the control group women not only had reduced depression by the end of the therapy period, but they also had reduced depression and cortisol levels during the postpartum period. Their newborns were also less likely to be born prematurely (4 vs 16\%) and with low birthweight ( 2 vs 10\%), and they had lower cortisol levels and performed better on the Brazelton Neonatal Behavioral Assessment habituation, orientation and motor scales.

The higher-than-national-average prematurity and low birthweight rates for the depressed mothers in this sample were not surprising given previous reports on a greater incidence of prematurity in depressed women $[5,6]$. The decreased depression and low back pain in the massaged women and their fewer prenatal complications, including a $75 \%$ lower prematurity rate and an $80 \%$ lower incidence of low birthweight in the massaged depressed women, were also not surprising given our previous finding of a lower prematurity rate in depressed women given pregnancy massage [2].

These findings were unique in that post partum depression and cortisol levels were decreased in the massaged women. The decrease in depression by week 32 of gestation in the massaged women persisted through the duration of pregnancy and the postpartum period. To have mothers less depressed during the neonatal period would likely enhance their bonding with their infants. Having more mature and more interactive newborns would also certainly enhance that process [7]. The fact that the newborns of the massaged mothers also had lower cortisol levels than the newborns of the control mothers may relate to the mothers' lower cortisol levels and their decreasing depression across pregnancy.

\section{Labor massage}

It is interesting that while labor is an anxiety-provoking experience, and while massage therapy has been noted to reduce anxiety in many studies, the focus of massage therapy labor studies has been almost exclusively on the alleviation of pain. In one study, massage was noted to lessen the pain intensity during phase 1 and 2 dilation, but there were no significant differences between the massage and control groups at phase 3 dilation [8].

In a study we conducted on labor pain, the pregnant women's partners massaged them during the first 15 min of every hour of labor [9]. The partners were given instructions on massaging the back and the legs (the areas of greatest pain) of the pregnant women from a side-lying position. The women experienced significantly less pain, and their labors were on average $3 \mathrm{~h}$ shorter with less need for medication (Figure 2).

\section{Potential underlying mechanisms for massage therapy effects}

Some suggest that massage effects can be explained by the 'gate theory' [10]. This is a kind of metaphor for the pain message traveling more slowly than the pressure message from massage (i.e., the stimulation of pressure receptors). Pressure messages travel more rapidly than pain messages, reaching the brain faster and closing the gate to the pain message. A more biological explanation of the gate theory is that cells in the dorsal horn of the spinal cord act like a switch between the nerve impulses from the different fibers. Pain is carried more slowly by the less insulated (unmyelinated) $\mathrm{C}$ neurons, whereas massage signals are carried more rapidly by the A neurons (myelinated) that close the gate to the $\mathrm{C}$ impulses and allow the $\mathrm{A}$ 
signals through. In this way, the massage stimulation (A fibers) closes the gate to the pain stimulus (C fibers).

An alternative underlying mechanism we have been exploring is that these effects are mediated by increased vagal activity. Vagal activity increases immediately after massage therapy sessions and across repeated sessions of massage therapy [11]. In these studies, significant increases in vagal activity occurred following massage therapy. This likely happens by the stimulation of pressure receptors that are innervated by vagal afferent fibers, which ultimately project to the limbic system, including hypothalamic structures involved in autonomic nervous system regulation and cortisol secretion.

These pathways are supported by several lines of evidence. First, anatomical studies indicate that baroreceptors, and to a lesser extent, mechanoreceptors under the skin (i.e., Pacinian corpuscles) are innervated by vagal afferent fibers projecting to the vagal nucleus of the solitary tract, the predominant source of afferent inputs to the efferent neurons of the nucleus ambiguous and the dorsal motor nucleus of the vagus [12]. Second, functional studies indicate that electrical vagal stimulation results in reduced cortisol responses in depressed patients [13]. Third, as already noted, we have recently shown that moderate pressure massage (but not light pressure massage) elicits a significant increase in vagal activity in both infants and adults [14]. Fourth, data collected across several studies by our group and others indicate that massage therapy decreases heart rate [15-17], lowers blood pressure [17-19] and reduces cortisol levels [20,21]. Last, a functional MRI study revealed that massage therapy increased cerebral blood flow across several brain regions involved in depression and stress regulation, including the amygdala and the hypothalamus [22], suggesting that massage therapy involves hypothalamic regulation of autonomic nervous system activity, cortisol secretion and limbic activity associated with emotion regulation.

Inverse relationships between vagal activity and cortisol levels have also been reported [23]. In addition, psychological stressors that reduce vagal activity have been noted to increase cortisol levels [24]. Increased vagal activity results in a slowing of physiology (decreased heart rate and blood pressure) and downregulation of cortisol [25]. Others have interpreted this relationship as vagal activity playing an inhibitory role in the regulation of allostatic systems [23]. As Thayer and Sternberg noted, the prefrontal cortex and amygdala are important CNS structures linked to the regulation of emotion and allostatic systems, including hypothalamicpituitary-adrenal axis function [23].

\section{Expert commentary}

Previous reports have found that depressed women have a greater incidence of prematurity and low birthweight rates $[5,6]$. In our studies, massage therapy has been effective during pregnancy [2-4]. Depressed women given massage therapy had fewer prenatal complications, including a 75\% lower prematurity rate and an $80 \%$ lower incidence of low birthweight [2]. They also reported decreased depression, anxiety and back pain. Surprisingly, postpartum depression and cortisol levels decreased in the massaged women. Their newborns also had lower cortisol levels than the newborns of the control mothers, which may relate to their mothers' lower cortisol levels and their decreasing depression across pregnancy. In addition, the newborns performed better on the Brazelton Neonatal Behavioral Assessment habituation, orientation and motor scales. With mothers less depressed during the neonatal period along with more mature and interactive newborns, the bonding process between them is enhanced [7].

In many studies massage therapy has been known for reducing anxiety. In the study we conducted on labor pain, the pregnant women's partners massaged their back and legs from a side-lying position during the first 15 min of every hour of labor [9]. The women experienced less pain, and their labors were on average $3 \mathrm{~h}$ shorter with less need for medication. 
Some credit the massage therapy effects with the gate theory [10]. This suggests that pressure messages travel more rapidly than pain messages, therefore reaching the brain faster and closing the gate to the pain message. Another underlying mechanism we have been exploring is that these massage therapy effects occur because vagal activity increases following massage therapy. In our previous studies, we have demonstrated that moderate-pressure massage and not light-pressure massage elicits a significant increase in vagal activity. According to a functional magnetic resonance imaging study, massage therapy increased cerebral flow in different brain regions involved in depression and stress regulation, including the amygdala and hypothalamus [22]. This implies that massage therapy engages hypothalamic regulation of autonomic nervous system activity, cortisol secretion and limbic activity related with emotional regulation. Psychological stressors that reduce vagal activity increase cortisol levels [24], but increased vagal activity decreases heart rate and blood pressure, as well as cortisol [25].

\section{Five-year view}

Like China, the USA may eventually have touch rooms in their hospitals where couples will learn the massage for both the pregnant women and the newborn. In time, the USA may come to have the low $1 \%$ rate of prematurity of China instead of the $14 \%$ rate it currently has. Additional research on underlying mechanisms for the massage effects will help to advance this field.

\section{Key issues}

- Massage therapy is the most common alternative therapy recommended during pregnancy.

- Massage therapy has been demonstrated to be effective during pregnancy. The women reported decreased depression, anxiety, and leg and back pain.

- Depressed pregnant women given the pregnancy massage experienced fewer prenatal complications.

- In our study on labor pain, the women receiving massage therapy experienced significantly less pain, and their labors were on average $3 \mathrm{~h}$ shorter with less need for medication.

- The most important finding was the lower incidence of prematurity and low birthweight in the massaged depressed women.

- Postpartum depression and cortisol levels were decreased in the massaged women. The newborns of the massaged mothers also had lower cortisol levels than the newborns of the control mothers, and performed better on the Brazelton Neonatal Behavioral Assessment habituation, orientation and motor scales. An underlying mechanism we have been exploring is that these effects are mediated by increased vagal activity.

\section{Acknowledgments}

Tiffany Field would like to thank her collaborators and Lissette Medina for assisting with this research, and the pregnant women who participated in her studies.

The studies discussed in this article were supported by NIH Senior Research Scientist Awards (MH 00331 and AT 01585) and an NIH Merit award (M 46586), NIH grants (AT1003 and HD 056036), and a March of Dimes grant (12FY03-48) to Tiffany Field and funding from the Johnson \& Johnson Pediatric Institute. 


\section{References}

Papers of special note have been highlighted as:

•• of considerable interest

1. Wang SM, Dezinno P, Fermo L, et al. Complementary and alternative medicine for low-back pain in pregnancy: a cross-sectional survey. J. Altern. Complement. Med 2005;11:459-464. [PubMed: 15992230]

2. Field T, Diego M, Hernandez-Reif M, Schanberg S, Kuhn C. Massage therapy effects on depressed pregnant women. J. Psychosom. Obstet. Gynaecol 2004;25:115-122. [PubMed: 15715034] •• In this study, immediately after the massage therapy sessions, the women reported lower levels of anxiety and depressed mood, and less leg and back pain. By the end of the study the massage group had higher dopamine and serotonin levels, and lower levels of cortisol and norepinephrine.

3. Field T, Diego M, Hernandez-Reif M, Deeds O, Fiqueiredo B. Pregnancy massage reduces prematurity, low birthweight and postpartum depression. Infant Behav. Dev 2009;32:454-460. [PubMed:

19646762] • In this study, the massage therapy group women versus the control group women not only had reduced depression by the end of the therapy period, but also had reduced depression and cortisol levels during the postpartum period. Their newborns were also less likely to be born prematurely and with low birthweight, and they had lower cortisol levels and performed better on the Brazelton Neonatal Behavioral Assessment habituation, orientation and motor scales.

4. Field T, Hernandez-Reif M, Hart S, et al. Pregnant women benefit from massage therapy. J. Psychosom. Obstet. Gynaecol 1999;20:31-38. [PubMed: 10212885] •• Showed decreased anxiety and stress hormones (norepinephrine) during pregnancy, and fewer obstetric and postnatal complications, including lower prematurity rates following pregnancy massage.

5. Oberlander TF, Warburton W, Misri S, Aghajanian J, Hertzman C. Neonatal outcomes after exposure to selective serotonin reuptake inhibitor antidepressants and maternal depression using population based linked health data. Arch. Gen. Psychiatry 2006;43:283-290.

6. Orr ST, James SA, Blackmore P. Maternal prenatal depressive symptoms and spontaneous preterm births among African American women in Baltimore, Maryland. Am. J. Epidemiol 2002;156:797802. [PubMed: 12396996]

7. Field T, Diego M, Hernandez-Reif M. Prenatal depression effects on the fetus and newborn: a review. Infant Behav. Dev 2006;29 •• Review of research on prenatal depression effects on the fetus and newborn suggests that they experience prenatal, perinatal and postnatal complications. Elevated prenatal maternal cortisol is the strongest predictor of these neonatal outcomes. Moderate pressure massage can alleviate these effects, including reducing prematurity.

8. Chang MY, Chen $\mathrm{CH}$, Huang KF. A comparison of massage effects on labor pain using the McGill Pain Questionnaire. J. Nurs. Res 2006;14:190-197. [PubMed: 16967401]

9. Field T, Hernandez-Reif M, Taylor S, Quintino O, Burman I. Labor pain is reduced by massage therapy. J. Psychosom. Obstet. Gynaecol 1997;18:286-291. [PubMed: 9443139] •• Massage therapy during the first $15 \mathrm{~min}$ of every hour of labor decreased anxiety and pain and the need for pain medication. In addition, the massaged mothers had shorter labor, a shorter hospital stay and less depressed mood.

10. Foster JM, Sweeney BP. The mechanisms of acupuncture analgesia. Br. J. Hosp. Med 1987;38:308312. [PubMed: 3315081]

11. Diego M, Field T, Hernandez-Reif M. Preterm infant massage consistently increases vagal activity and gastric mobility. Acta Paediatr 2007;96:1588-1591. [PubMed: 17888059] • Massaged infants exhibited consistent short-term increases in vagal activity and gastric motility on both the first and the last days of the 5-day study that were associated with weight gain during the 5-day treatment period.

12. Kandel, E.; Schwartz, JH.; Jessell, TM. Principles of Neural Science. 4th Edition. McGraw-Hill; NY, USA: 2000.

13. O'Keane V, Dinan TG, Scott L, Corcoran C. Changes in hypothalamic-pituitary-adrenal axis measures after vagus nerve stimulation therapy in chronic depression. Biol. Psychiatry 2005;58:963968. [PubMed: 16005439] 
14. Field T, Diego M. Vagal activity, early growth and emotional development. Infant Behav. Dev 2008;31:361-373. [PubMed: 18295898] •• A review of the research on infant vagal tone suggests that vagal activity is associated with both infant growth and infant socio-emotional development. These studies highlight the relationship between vagal activity and the social behaviors of attentiveness, facial expressions and vocalizations.

15. Diego MA, Field T, Sanders C, Hernandez-Reif M. Massage therapy of moderate and light pressure and vibrator effects on EEG and heart rate. Int. J. Neurosci 2004;114:31-45. [PubMed: 14660065] - Anxiety scores decreased for all groups, but the moderate pressure massage group reported the greatest decrease in stress.

16. Kaye AD, Kaye AJ, Swinford J, et al. The effects of deep-tissue massage therapy on blood pressure and heart rate. J. Altern. Complement. Med 2008;14:125-128. [PubMed: 18315516]

17. Kubsch SM, Neveau T, Vandertie K. Effect of cutaneous stimulation on pain reduction in emergency department patients. Complement. Ther. Nurs. Midwifery 2000;6:25-32. [PubMed: 11033650]

18. Ahles TA, Tope DM, Pinkson B, et al. Massage therapy for patients undergoing autologous bone marrow transplantation. J. Pain Symptom Manage 1999;18:157-163. [PubMed: 10517036]

19. Hernandez-Reif M, Field T, Krasnegor J, Theakston H, Hossain Z, Burman I. High blood pressure and associated symptoms were reduced by massage therapy. J. Bodyw. Mov. Ther 2000;4:31-38.

20. Field T, Hernandez-Reif M, Diego M, Schanberg S, Kuhn C. Cortisol decreases and serotonin and dopamine increase following massage therapy. Int. J. Neorosci 2005;115:1397-1413.

21. Kim MS, Cho KS, Woo H, Kim JH. Effects of hand massage on anxiety in cataract surgery using local anesthesia. J. Cataract Refract. Surg 2001;27:884-890. [PubMed: 11408136]

22. Ouchi Y, Kanno T, Okada H, et al. Changes in cerebral blood flow under the prone condition with and without massage. Neurosci. Lett 2006;407:131-135. [PubMed: 16973270]

23. Thayer JF, Sternberg E. Beyond heart rate variability: vagal regulation of allostatic systems. Ann. NY Acad. Sci 2006;1088:361-372. [PubMed: 17192580]

24. Spangler G. Psychological and physiological responses during an exam and their relation to personality characteristics. Psychoneuroendocrinology 1997;22:423-441. [PubMed: 9364621]

25. Porges SW. The polyvagal theory: phylogenetic substrates of a social nervous system. Psychoneuroendocrinology 2001;23:837-861. [PubMed: 9924740] 


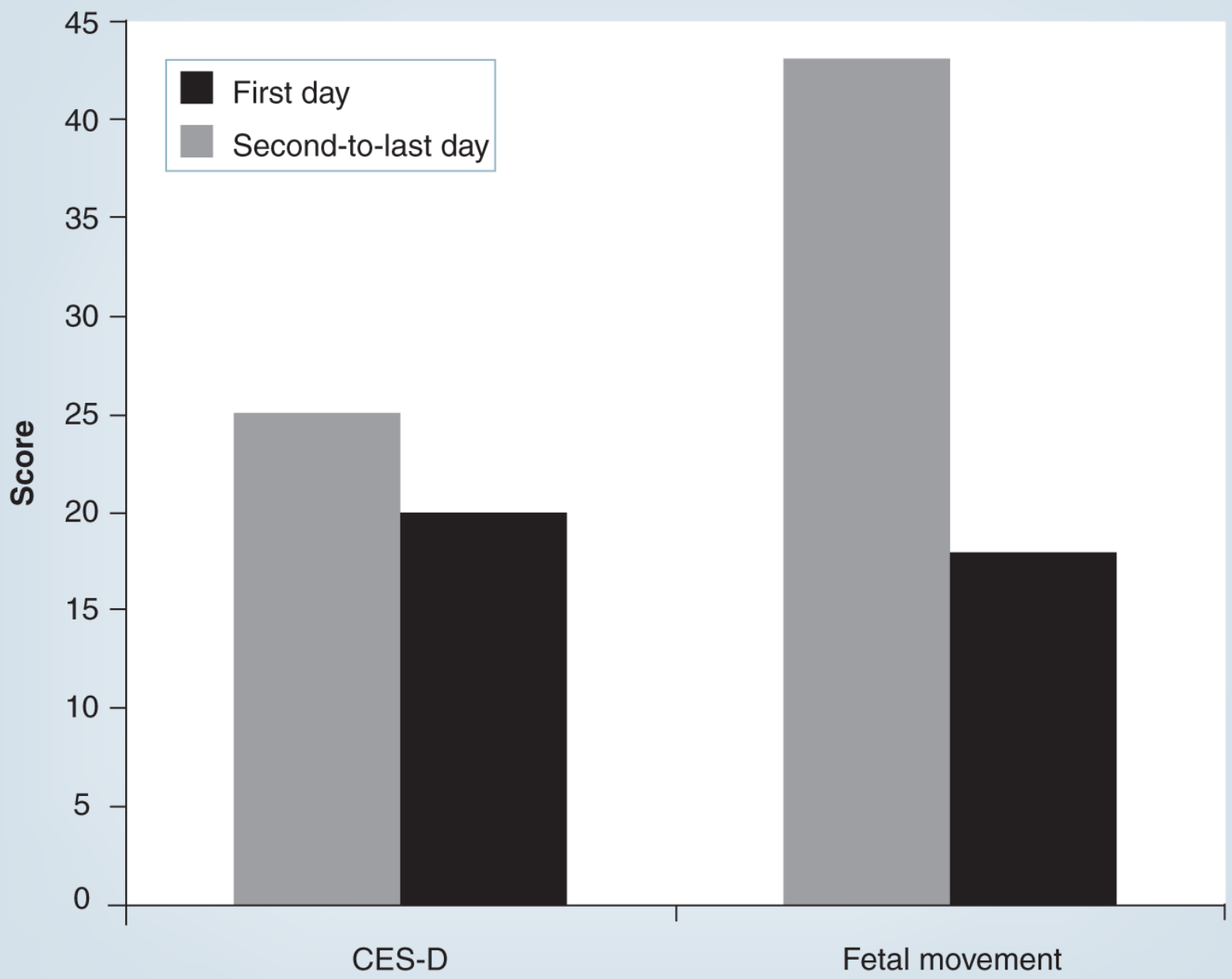

Figure 1. Mean Center for Epidemiological Studies Depression scores and fetal movement scores on first day (black) and second to last day (gray) of pregnancy massage study CES-D: Center for Epidemiological Studies Depression Score.

Data from [2]. 


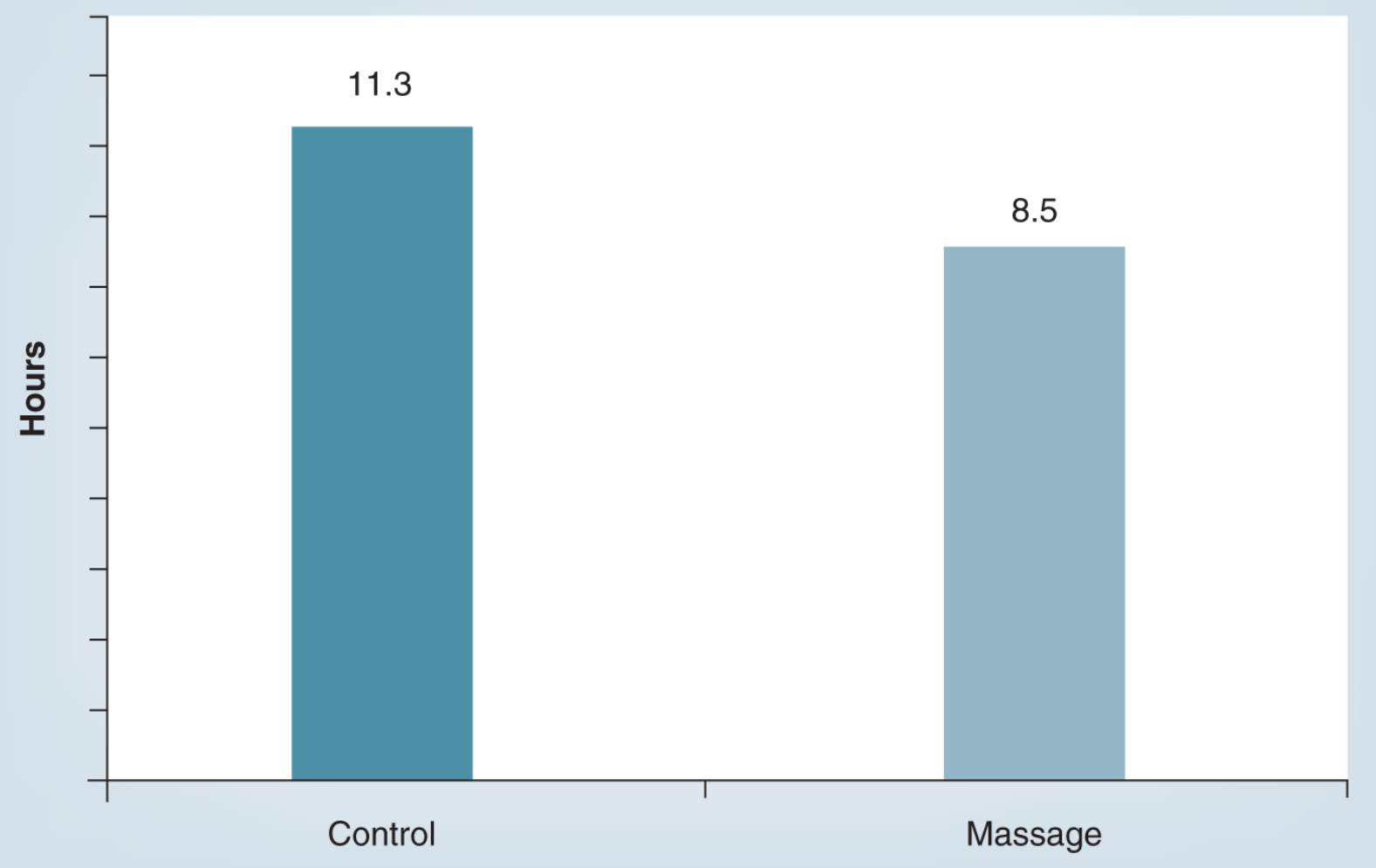

Figure 2. Mean number hours of labor in control and massage groups Data from [9]. 